\title{
Human Behavior and Deception Detection
}

Mark G. Frank, University at Buffalo, State University of New York

Maureen O'Sullivan, University of San Francisco

Melissa A. Menasco, University at Buffalo, State University of New York

\section{Keywords}

Behavior; Cognition: Deception; Detection; Emotion; Judgment; Lying; Malfeasance; Memory.

\begin{abstract}
Human intelligence is the key to stopping terrorism, and therefore it is essential to know when the information obtained is false. This chapter briefly outlines the research on behavioral clues to deception, as well as research on people's abilities to spot deception once it has happened. We find that there is no clue or clue pattern that is specific to deception, although there are clues specific to emotion and cognition. In general, behavioral clues are only limited in their abilities to identify deception and that there are still behavioral measurement issues that may plague research on deception. Moreover, a closer examination of the laboratory research suggests many research studies are not relevant to security contexts, thus the research literature may underestimate the usefulness of behavioral information - particularly for the utility of identifying emotional and cognitive states. We also find that most people, unaided by technology, cannot detect lies from behavior information, but that some groups do show significantly higher levels of accuracy - although more research is needed to understand why. We conclude that more directed interaction with scientists and practitioners - in both lab work and in the real world, in creating real world databases, in identifying base rates for malfeasant behavior in security settings, in optimizing training and in identifying pre existing excellence within security organizations can more rapidly capitalize on the usefulness of behavioral information in security settings.
\end{abstract}

Terrorism at its core is a human endeavor. Human beings cultivate what they hate, plan and then execute terrorist attacks. Thus, any information that can aid the intelligence or security officer to weigh the veracity of the information he or she obtains from suspected terrorists or those harboring them would help prevent attacks. This would then not only add another layer to force protection but would facilitate future intelligence gathering. Yet the face to face gathering of information through suspected terrorists, informants, or witnesses is replete with obstacles that affect its accuracy such as the well documented shortcomings of human memory, honest differences of opinion, as well as what is the focus of this chapter - outright deception [1].

The evidence suggests that in day to day life most lies are betrayed by factors or circumstances surrounding the lie, and not by behavior [2]. However, there are 
times when demeanor is all a Homeland security agent has at his or her disposal to detect someone who is lying about his or her current actions or future intent. Because a lie involves a deliberate, conscious behavior, we can speculate that this effort may leave some trace, sign, or signal that may betray that lie. What interests the scientist, as well as society at large, is a) Are there clues perceptible to the unaided eye that can reliably discriminate between liars and truth tellers; $b$ ) Do these clues consistently predict deception across time, types of lies, different situations, and cultures?; and if a) and b) are true, then c) How well can our counter-terrorism professionals can make these judgments, and can they do this in real time, with or without technological assistance?

\section{Scientific overview - behavioral signs of deception.}

To date no researcher has documented a "Pinocchio response;" that is, a behavior or pattern of behaviors that in all people, across all situations, is specific to deception (e.g., [3]). All the behaviors identified and examined by researchers to date can occur for reasons unrelated to deception. Generally speaking, the research on detecting lies from behavior suggests that two broad families of behavioral clues are likely to occur when someone is lying - clues related to liars' memory and thinking about what they are saying (cognitive clues), and clues related to liar's feelings and feelings about deception (emotional clues) $[3,4,5,6$, $7,8]$.

\section{Cognitive clues.}

A lie conceals, fabricates, or distorts information; this involves additional mental effort. The liar must think harder than a truth teller to cover up, create events that have not happened, or to describe events in a way to allow multiple interpretations. Additional mental effort is not solely the domain of the outright liar, however; a person who must tell an uncomfortable truth to another will also engage in additional mental effort to come up with the proper phrasing while simultaneously reducing the potential negative emotional reaction of the other. This extra effort tends to manifest itself with longer speech latencies, increased speech disturbances, less plausible content, less verbal and vocal involvement, less talking time, more repeated words and phrases, and so forth [9]. Research has also shown that some nonverbal behaviors change as a result of this mental effort. For example, illustrators - hand or head movements that accompany speech, and are considered by many to be a part of speech (e.g., [10]) - will decrease when lying compared to telling the truth $[11,12]$.

Another way in which cognition is involved in telling a lie is through identification of naturalistic memory characteristics. This means that experienced events have memory qualities that are apparent upon description that are different from events that have not been experienced (the "Undeutsch hypothesis" [13]). Events that were not actually experienced feature more ambivalence, have fewer details, a poorer logical structure, less plausiblity, more negative statements and 
are less embedded in context. Liars are also less likely to admit lack of memory, and have less spontaneous corrections (reviewed by [8, 9]), and may use more negative emotion words, and fewer self and other references [14]. Mental effort clues seem to occur more in the delivery of the lie, whereas memory recall clues tend to occur in the content of the lie.

We note that not all lies will tax mental effort; for example, it is much less mentally taxing to answer a close ended question like "Did you pack your own bags?" with a yes or no than to answer an open ended "What do you intend to do on your trip?" Moreover, a clever liar can appear more persuasive if he or she substitutes an actual experienced event as their alibi rather than creating an entirely new event. This may be why a recent general review paper [9] found consistent non-homogenous effect sizes for these mental effort and memory based cues across the studies they reviewed, as the particular paradigms used by researchers varied greatly in the extent to which the lies that were studied mentally taxed the liars.

\section{Emotional clues.}

Lies can also generate emotions, ranging from the excitement and pleasure of "pulling the wool over someone's eyes" to fear of getting caught to feelings of guilt [4]. Darwin [15] first suggested that emotions tend to manifest themselves in the facial expressions, as well as in the voice tones, and that these can be reliable enough to accurately identify emotional states. Research has since shown that for some expressions - e.g., anger, contempt, disgust, fear, happy, sadness/distress, or surprise -cultures throughout the planet recognize and express these emotions in both the face and voice similarly [16]. To the extent that a lie features higher stakes for getting caught, we would expect to see more of these signs of emotion in liars compared to truth tellers. If the lie is a polite lie that people tell often and effortlessly, there would be less emotion involved (e.g., [17]). Meta-analytic studies suggest that liars do appear more nervous than truth tellers, with less facial pleasantness, higher vocal tension, higher vocal pitch, greater pupil dilation and fidgeting [9]. If the lie itself is about emotions - e.g., telling someone that one feels calm, when in fact one is nervous - the research shows that signs of the truly felt emotion appear in the face and voice despite attempts to conceal, although these signs are often subtle and brief $[18,19]$.

\section{Measurement issues.}

One issue in measuring lie signs is to make clear what is meant by the terms cognition and emotion. For example, in deception research the term arousal is used interchangeably with emotion, but often refers to many different phenomena: an orienting response (e.g., [20]), an expression of fear (e.g., [21]), a more indeterminate affect somewhere between arousal and emotion (e.g., [22]; see also discussion by Waid \& Orne [23]), as well as physiological states as different as stress, anxiety, embarrassment and even anger [24]. 
A second issue in measuring lie signs is to clarify the level of detail of measurement as well as to specify why that level of detail may or may not correlate with lying [25]. Many meta-analyses of behavioral deception clues report insignificant effect sizes, but the variance among effect is not homogenous (e.g., [3; 9, 26; 27; 28]). For example, some studies investigated behavior at the most elemental physical units of measurement such as counting the movements in the hands, feet, arms, legs, torso, eye movements, eye blinks, pupil dilation, lip pressing, brow lowering or raising, lip corner puller (smiling), fundamental frequency, amplitude, pauses, filled pauses, response latency, speech rate, length of response, connector words, unique words, self-references, and so forth. Other studies investigated behavior at the most elemental psychological meaning units of measurement. Some of these included manipulators - which involve touching, rubbing, etc., of various body parts - which could be composed of a number of hand, finger, arm movements, but which were scored for theoretical rather than merely descriptive reasons. Other psychologically meaningful units of measurement include illustrators, which accompany speech to help keep the rhythm of the speech, emphasize a word, show direction of thought, etc., or emblems, which are gestures that have a speech equivalent, such as a head nod meaning "yes", or a shrug meaning "I'm not sure", or facial emblems such as winking. The psychological meaning units might also include vocal tension, speech disturbances, negative statements, contextual embedding, unusual details, logical structure, unexpected complications, superfluous details, self doubt, and so forth. Finally, other studies investigated behavior at the most interpretative/impressionistic unit level, which are further unarticulated composites of the physical and the psychological meaning units described earlier. Some of these behaviors impressionistic variables include fidgeting, involvement, body animation, posture, facial pleasantness, expressiveness, vocal immediacy and involvement; and spoken uncertainty; plausibility, cognitive complexity (again, see review by [9]). The problem of course is that as one moves from physical to impressionistic measures, it would seem become harder to make those judgments reliably. This is not always the case though; e.g., the term "smile" has rarely been defined in research reports, yet independent coders are typically above .90 reliability when coding smiles (see [29] for a review). Although research suggests that people can be more accurate when they employ indirect inferences to deception (e.g., Does the person have to think hard? [30]), 'gut' impressions tend to be uncorrelated with accuracy [26]. This suggests that we must be cautious about clues at the impressionistic level, and that it may be more productive to study them at their psychological level where they might be more meaningful to understanding deception.

Prognosis on generalizability of deception findings across time, lies, situations, and cultures.

It is safe to conclude that although there are some clues that betray a lie at rates greater than chance, none of them are exclusive to deception. This conclusion 
applies to machine based physiological approaches as well. However, the origins of these signs - mental effort, memory, and emotion - are universal. This suggests that if the context in which the information is gathered is controlled, and designed to differentially affect liars and truth tellers, it would increase greatly the chances of being able to distinguish people with deceptive intent from those with truthful intent. Polygraph examination has done this by controlling their question style to improve hit rates, but to date this has not been done systematically in behavioral studies. Thus its effects are unknown, but we can speculate based upon what we know about normal, truthful human behavior. If the lie is of no significance to the person, with no costs for getting caught, and involves a simple yes or no answer, odds are there will not be many clues to distinguish the liar and the truth teller. If the situation has significance to the person, there are consequences for getting caught, and the person is required to recount an event in an open ended question, then we would expect more clues to surface that would distinguish the liar from the truth teller. This may be a curvilinear relationship; a situation of extraordinary high mental effort and emotion - e.g., one in which a person is being beaten, screamed at, and threatened with execution - will generate all the "lie clues" described earlier, but equally in liar and truth teller. Nonetheless, information about mental effort, experienced memory, and emotion can be very useful clues to Homeland Security personnel to identify behavioral "hot spots" [4] that can provide information about issues of importance to the subject. A counter-terrorism Intelligence officer who knows when a subject is feeling an emotion or thinking hard can know what topics to pursue or avoid in an interview, whether the subject is fabricating, concealing information, or merely feeling uncomfortable with the topic, although truthful.

\section{Scientific overview - abilities to spot liars.}

Research over the past 30 years suggests that the average person is slightly statistically better than chance at identifying deception, but not practically better. The most recent review of over 100 studies has shown that when chance accuracy is $50 \%$, the average person is approximately $54 \%$ accurate [31]. There are a number of reasons for this poor ability; among them poor feedback in daily life (i.e, a person only knows about the lies they have caught); the general tendency for people to believe others until proven otherwise (ie, a 'truth bias'; [32]), and especially a faulty understanding of what liars actually look like (ie, the difference between people's perceived clues to lying compared to the actual clues; [26]).

\section{General abilities of specialized groups.}

Most of the studies reviewed were laboratory based and involved observers judging strangers. But similar results are found even when the liars and truth tellers are known to the observers (also reviewed by [31]. If the lies being told are low stakes, so that little emotion is aroused and the lie can be told without much extra cognitive effort, there may be few clues available on which to base a 
judgment. But even studies of high stakes lies, in which both liars and truth tellers are highly motivated to be successful, suggest an accuracy level that is not much different than chance.

Research that examined unselected professionals involved in security settings police, federal agents, and so forth - have typically found that they too are not any more accurate in their abilities to spot deception than laypeople (e.g., [27, $33,34,35,36])$. However, within these studies there have been a handful of groups that have performed better than $60 \%$ accurate on both lies and truths, and what these groups are doing might be informative for Homeland Security applications. The first group identified was a group of Secret Service agents who not only were superior, as a group, in detecting lies about one's emotions, but those who were more accurate were more likely to report using nonverbal clues than those who were less accurate. The authors [33] speculated that the Secret Service agents were more accurate than the other groups because they were trained in scanning crowds for nonverbal behaviors that did not fit, and they also dealt with assassination threats, many of which were made by mentally ill individuals. Unlike most police officers whose assumption of guilt in suspects is high [37], reflecting the experience of their daily work, Secret Service agents interviewed suspects where they knew the base rate of true death threats was low. The second set of groups identified included forensic psychologists, federal judges, selected federal law enforcement officers and a group of sheriffs [34]. A commonality among these groups seemed to be their very high motivation to improve their lie detecting skills. A third set of groups identified were police officers examining real life lies, who showed $65 \%$ overall accuracy in detecting lies and truths [38].

\section{Individual differences.}

As with any ability, research suggests that some people are better able to detect deception that others in high stake lies (e.g., [39]); this skill does not seem to translate to lower stake lies [32]. One element of better skill in higher stake settings is the ability to judge micro-momentary displays of emotion [33, 39]. Other groups who showed better than 60\% accuracy included people with left hemisphere brain lesions that prevented them from comprehending speech [40], and those subjects who scored higher on a test of knowledge of clues to deceit were also more accurate than those who did not [41]. A different approach has been to identify individuals who obtain high scores on lie detection tests and studying them in detail [42]. After testing more than 12,000 people using a sequential testing protocol involving three different lie detection accuracy measures, O'Sullivan and Ekman identified 29 highly accurate individuals. These individuals had a kind of genius with respect to the observation of verbal and nonverbal clues, but since genius often connotes academic intelligence, the expert lie detectors were labeled "truth wizards" to suggest their special talent. Although this term is unfortunate in mistakenly suggesting that their abilities are due to magic rather than talent and practice, the term does reflect the rarity of their abilities. One of the first findings of the Wizard Project was a profession 
specific sensitivity to certain kinds of lies. About $1 / 3$ of the wizards were highly accurate on all three of the tests used. Another third did very well on two of the tests, but not on the third, in which people lied or told the truth about whether they had stolen money. Nearly all of these wizards were therapists who had little, if any, experience with lies about crime. On the other hand, the remaining third of the wizards were law enforcement personnel - police and lawyers - who did very well on the crime lie detection test, but not on a test in which people lied or told the truth about their feelings. Compared with a matched control group, expert lie detectors are more likely than controls to attend to a wide array of nonverbal behaviors and to be more consciously aware of inconsistencies between verbal and non-behaviors. Although expert lie detectors make almost instantaneous judgments about the kind of person they are observing, they are also more cautious than controls about reaching a final decision about truthfulness.

\section{Critical needs analysis.}

Research on human behavior and deception detection can make a useful contribution to Homeland Security needs as long as scientists and practitioners understand what it is they are observing -signs of thinking, signs of feeling. This rule applies to automated approaches that measure physiology as well. Even with this limitation, training in behavioral hot spot recognition may make security personnel better at spotting those with malfeasant intent. Other critical needs include:

\section{More relevant laboratory paradigms and subjects.}

We must recognize that general meta-analyses of the research literature, although useful, are limited in their applicability to security contexts, since such analyses tend to combine studies that feature lies told with few stakes and cognitive demands with those with higher stakes and stronger cognitive demands. Thus, we should be more selective about which studies to examine for clues that may be useful or relevant to security contexts. This also means it is important for scientists to develop research paradigms that more closely mirror the real life contexts in which security personnel work. Although laboratory settings are not as powerful as real world settings, high stake laboratory deception situations can provide insights with the best chance of applicability. Consistent with this approach, two current airport security techniques capitalize on behaviors identified by research studies on stress, with anecdotal success (ie, TSA's Screening Passengers by Observation Techniques and the MA State Police Behavioral Assessment System). One way to facilitate this type of progress is to have Homeland Security personnel advise laboratory research, as well as allow researchers to spend on-the-job time with Security personnel. We believe pairing the researchers and practitioners would eventually result in calls for laboratory studies featuring higher stakes to the liars, different subject populations beyond US/Europeans (as research suggests that people can detect 
deception in other cultures at rates greater than chance; $[43,44])$, and differing interview lengths such as examining shorter interviews (i.e, a 30-90 second security screening) and longer interviews (i.e., a 1-4 hour intelligence interview).

\section{Examination and creation of real world databases.}

There have been very few studies of real world deception (e.g., [38]), yet the technological capability exists to create many more. The biggest problem with real world data is determining the ground truth (was the person really lying, or did they truly believe what they just stated?). Estimating ground truth - as compared to knowing ground truth - will slow down the identification of any patterns or systems. Clear criteria must be established a priori to determine this ground truth. For example, confessions of malfeasance are a good criterion, but false confessions do happen. Catching someone with contraband (i.e., a 'hit') is also a good criterion, but occasionally the person may be truthful when he or she states that someone must have snuck it into their luggage. Moreover, academics should advise on the capture and recording of these databases, to insure that the materials are able to be examined by the widest number of researchers and research approaches. For example, most of the police interview video we have seen is of such poor quality that we cannot analyze facial expressions in any detail. It is only when these databases are combined with the laboratory work that we can more sharply identify behaviors or behavioral patterns that will increase the chances of catching those with malfeasant intent. To optimally use this information though, we must also examine in detail known cases of false negatives and false positives as well as correct hits to determine why mistakes were made in these judgments.

\section{Ground truth baserates.}

Security personnel do not know is the base rates for malfeasance in their settings. Although it may be logistically impossible to hand search every piece of hand luggage in a busy airport, or follow every investigative lead, it would be essential to know this base rate in order to ascertain the effectiveness of any new behavioral observational technique. This would also permit more useful costbenefit analyses of various levels of security and training. A less satisfying but still useful way to ascertain effectiveness is to compare hit rates for contraband for those using various behavioral observation techniques with those who are stopped randomly (as long as the day of the week and time of the day/year are scientifically controlled).

\section{Optimizing Training.}

The most recent meta-analysis of the research literature on training people to improve deception detection from behavior has shown that across over 2,000 subjects, there was a modest effect for training, despite the use of substandard training techniques [45]. This obviously suggests that better training techniques will yield larger improvements in people's abilities to sort out truth from lie. One 
training change would be to train on behavioral clues that are derived from similar situations and supported by research. For example, one study trained research subjects to recognize a set of behavioral clues that are believed to be indicative of deception, and are often taught to law enforcement personnel as signs of deception, although many of these signs are not supported by the scientific literature [46]. This study reported a 10\% decrease in accuracy for the groups receiving such training. Therefore, the first step in adequate training is to identify what information is useful for training (see above). The second step is to determine the most effective way to deliver that information. For example, what is the training duration that maximizes comprehension - one full day, 3 full days, or more? Should it be done in a group or self study? Does it need simple repetition, or more creative approaches, and how many training items are needed? Does it need to be reinforced at particular intervals? How many clues should be taught i.e., at what point do you overwhelm trainees? How do you train in such as way as to improve accuracy without over inflating confidence? These are just a few of the questions with unknown answers.

\section{Identifying Excellence.}

Another critical need is to identify who within relevant organizations shows signs of excellence, through their higher hit rates or whatever other clear criteria can be applied. This strategy is similar to the strategy of the "wizards" study [42]. One caution is that to date, most testing material will be laboratory experiment based, and the generalizability of that information to real world contexts is not perfect. An examination of the convergent validity of laboratory tests of deception detection and other more naturalistic approach measures (peer ratings, field observations in airports or other points of entry with accuracy determined by the rate of contraband "hits" by individuals compared to random selection) would be a great start.

\section{Future research directions.}

The aforementioned critical needs suggest several research questions, but by no means is that section comprehensive. As we peer into the future, there is much work to do. A partial list of future directions suggests we should:

- Examine the role of technology in facilitating behavioral observation. A number of computer vision algorithms are now available that can aid observation, such as recognizing emotional expressions in the face (e.g., [47]). What is unknown is how robust these algorithms are in real world contexts. What is also unknown is how best to combine technological observation of behavior with human judgment. Would there be a tendency for humans to over rely upon the technology over time?

- Identify the optimal environmental set up for surveillance, whether with technology or the unaided eye. This includes proxemic placement of 
tables, lines, stanchions, other individuals, and so forth. One goal would be to create an environment that would reduce the typical stress felt by the normal traveler, which would hopefully increase the salience of any sign of stress exhibited by the malfeasant to increase the chances it can be observed.

- Identify optimal interaction style between security agents and the public. One can aggressively question and threaten travelers, but that might render behavioral observation useless due to the overall stress engendered. A rapport building approach (e.g., [48]) might be better, but this needs more research.

- Identify the optimal interview style. Phrasing of questions is important in obtaining information, but this has not been researched in the open literature. Small changes in phrasing - e.g., open versus close ended might add to the additional cognitive burden of the liar and thus could be useful. The order of questions will also be important, as well whether one should make a direct accusation. But only additional research will tell.

- Identify the optimal way to combine behavioral clues. Research tends to examine individual behavioral clues to ascertain their effectiveness, yet more modern neural network and machine learning approaches may be successful in identifying patterns and combinations of behaviors that better predict deception in particular contexts.

- Identify the presence of countermeasures. An inevitable side effect of the release of any information about what behaviors are being examined by security officers to identify riskier individuals in security settings is that this information will find its way onto the internet or other public forum. This means a potential terrorist can learn what to do and what not to do in order to escape further scrutiny. The problem is that we don't know yet whether one can conceal all their behaviors in these real life contexts. Moreover, some of these behaviors, like emotional behavior, is more involuntary [16] and should be harder to conceal that more voluntary behavior like word choice. Thus it remains an open question as to whether a potential terrorist can countermeasure all of the critical behaviors.

Space limitations preclude an exhaustive list of needs, future directions, and research. In general, the research suggests that there are limited clues that are useful to sorting out liars and truth tellers, but most people cannot spot them. However, a closer examination of this literature suggests that some behavioral clues can be useful to security personnel, and some people can spot these clues well. We feel that it may be ultimately most productive to expand our thinking about behavioral clues to deceit to include thinking about behavioral clues to a person's reality - clues that someone is recounting a true memory, is thinking hard, or is having an emotion they wish to hide. This would enable a security officer to make the most accurate inference about the inner state of the person they are observing, which, when combined with better interaction and interviewing techniques, would enable them to better infer the real reasons for this inner state, be it intending us harm, telling a lie, or telling the truth. 


\section{References}

1. Haugaard, J. J. \& Repucci, N. D. (1992). Children and the truth. In S. J. Ceci, M. DeSimone-Leichtman, and M.E. Putnick (Eds) Cognitive and social factors in early deception. Hillsdale, NJ: Erlbaum.

2. Park, H.S., Levine, T. R., McCornack, S. A., Morrison, K., \& Ferrar, M. (2002). How people really detect lies. Communication Monographs, 69, 144-157.

3. Zuckerman, M., DePaulo, B. M., \& Rosenthal, R. (1981). Verbal and nonverbal communication of deception. In L. Berkowitz (Ed.) Advances in experimental social psychology. Vol 14, (pp. 1-59). San Diego, CA: Academic Press.

4. Ekman, P. (1985/2001). Telling lies. New York: Norton.

5. Ekman, P., \& Frank, M. G. (1993). Lies that fail. In M. Lewis \& C. Saarni (Eds.) Lying and deception in everyday life. (pp 184-200). New York: Guilford Press.

6. Hocking, J. E., \& Leathers, D. G. (1980). Nonverbal indicators of deception: A new theoretical perspective. Communication monographs, 47, 119-131.

7. Knapp, M. L., \& Comadena, M. E. (1979). Telling it like it isn't: A review of theory and research on deceptive communication. Human Communication Research, 5, 270-285.

8. Yuille, J. C. (Ed.) (1989). Credibility Assessment. Dordrecht, Netherlands: Kluwer

9. DePaulo, B. M., Lindsay, J. J., Malone, B. E., Muhlenbruck, L., Charlton, K., \& Cooper, H. (2003). Cues to deception. Psychological Bulletin, 129, 74112.

10. McNeill, D. (1992). Hand and Mind. What Gestures Reveal about Thought. Chicago: Chicago University. Press.

11. Ekman, P., \& Friesen, W. V. (1972). Hand movements. Journal of Communication, 22, 353-374.

12. Vrij, A. (1995). Behavioral correlates of deception in a simulated police interview. The Journal of Psychology, 129, 15-28.

13. Undeutsch, U. (1967). Beurteilung der Glaubhaftigkeit von Aussagen. In U. Undeutsch (Ed.), Handbuch derPsychologie. Bd. II: Forensische Psychologie (pp. 26-181 ). Goettingen, Verlag fur Psychologie.

14. Newman, M. L, Pennebaker, J. W., Berry, D. S., \& Richards, J. M. (2003). Lying Words: Predicting Deception From Linguistic Styles. Personality and Social Psychology Bulletin, 29, 665-675.

15. Darwin, C. (1872/1998). The expression of the emotions in man and animals. New York: Oxford. (3 ${ }^{\text {rd }}$ Edition, w/ commentaries by Paul Ekman).

16. Ekman, P. (2003). Emotions revealed. New York: Henry Holt.

17. DePaulo, B. M., Kashy, D. A., Kirkendol, S.E., Wyer, M. M., \& Epstein, J.A. (1996). Lying in everyday life. Journal of Personality and Social Psychology, 70, 979-995.

18. Ekman, P., Friesen, W. V., \& O'Sullivan, M. (1988). Smiles when lying. Journal of Personality and Social Psychology, 54, 414-420. 
19. Ekman, P., O'Sullivan, M., Friesen, W. V., \& Scherer, K. (1991). Invited article: Face, voice, and body in detecting deceit. Journal of Nonverbal Behavior, 15, 125-135.

20. deTurck, M. A., \& Miller, G. R. (1985). Deception and arousal: Isolating the behavioral correlates of deception. Human Communication Research, 12, 181-201.

21. Frank, M. G. (1989). Human lie detection ability as a function of the liar's motivation. Unpublished doctoral dissertation, Cornell University, Ithaca, NY.

22. Burgoon, J. E., \& Buller, D. B. (1994). Interpersonal deception: III. Effects of deceit on perceived communication and nonverbal behavior dynamics. Journal of Nonverbal Behavior, 18, 155-184

23. Waid, W. M., \& Orne, M. T. (1982). The physiological detection of deception. American Scientist, 70, 402-409.

24. Steinbrook, R.(1992). The polygraph test: A flawed diagnostic method. New England Journal of Medicine, 327, 122-123.

25. Frank, M. G. (2005). Research methods in detecting deception research. In J. Harrigan, K. Scherer, \& R. Rosenthal (Eds.). Handbook of Nonverbal Behavior Research. London: Oxford. (pp 341-368).

26. DePaulo, B.M., Stone, J., \& Lassiter, D. (1985). Deceiving and detecting deceit. In B. R. Schlenker (Ed.) The self and social life. (pp.323-355).

27. Vrij, A. (2000). Detecting lies and deceit: The psychology of lying and the implications for professional practice. Chichester: John Wiley.

28. Zuckerman, M. \& Driver, R. E. (1985). Telling lies: Verbal and nonverbal correlates of deception. In W. A. Siegman \& S. Feldstein (Eds.)

Multichannel integration of nonverbal behavior. (pp. 129-147). Hillsdale, NJ: Erlbaum.

29. Frank, M.G. (2003). Smiles, lies, and emotion. In M. Abel (Ed). The smile: Forms, functions, and consequences. New York: The Edwin Mellen Press. (pp 15-43).

30. Vrij A.; Edward K.; \& Bull R. (2001). Police officers' ability to detect deceit: The benefit of indirect deception detection measures. Legal and Criminological Psychology, 6, 185-196

31. Bond, C.F, Jr., \& DePaulo, B. M. (2006). Accuracy of deception judgments. Personality and Social Psychology Review, 10, 214-234.

32. DePaulo, B. M., \& Rosenthal, R. (1979). Telling lies. Journal of Personality and Social Psychology, 37, 1713-1722.

33. Ekman, P., \& O'Sullivan, M. (1991). Who can catch a liar? American Psychologist, 46, 913-920.

34. Ekman, P., O'Sullivan, M., \& Frank, M.G. (1999). A few can catch a liar. Psychological Science, 10, 263-266

35. DePaulo, B.M., \& Pfeifer, R.L. (1986). On-the-job experience and skill at detecting deception. Journal of Applied Social Psychology , 16 , 249-267.

36. Kraut, R. E., \& Poe, D. (1980). Behavioral roots of person perception: The deception judgments of customs inspectors and laymen. Journal of Personality and Social Psychology, 39, 784-798. 
37. Meissner, C. A. \& Kassin, S. M. (2002). :"He's guilty!": Investigator bias in judgments of truth and deception. Law and Human Behavior, 26, 469480.

38. Mann, S., Vrij, A., \& Bull, R. (2004). Detecting true lies: Police officers' abilities to detect suspects' lies. Journal of Applied Psychology, 89, 137149.

39. Frank, M. G., \& Ekman, P. (1997). The ability to detect deceit generalizes across different types of high stake lies. Journal of Personality and Social Psychology, 72, 1429-1439.

40. Etcoff, N.L., Ekman, P., Magee, J.J., \& Frank, M.G. (2000). Superior lie detection associated with language loss. Nature; 405 (11 May), 139-139.

41. Forrest, J.A., Feldman, R. S., \& Tyler, J. M. (2004). When accurate beliefs lead to better lie detection. Journal of Applied Social Psychology, 34, 764780.

42. O'Sullivan, M.,\& Ekman, P. (2004). The wizards of deception detection. In Granhag, P.A., \& Stromwell, L. (Eds.), The Detection of Deception in Forensic Contexts (pp. 269-286). Cambridge, UK: Cambridge University Press.

43. Bond, C. F. Jr; Atoum, A. O. (2000). International deception. Personality \& Social Psychology Bulletin, 26, 385-395

44. Bond, C. F; Omar, A; Mahmoud, A; \& Bonser, R.N. (1990). Lie detection across cultures. Journal of Nonverbal Behavior, 14, 189-204.

45. Frank, M.G., \& Feeley, T.H. (2003). To catch a liar: Challenges for research in lie detection training. Journal of Applied Communication Research, 31, 58-75.

46. Kassin, S.M; \& Fong, C.T. (1999). "I'm innocent!": Effects of training on judgments of truth and deception in the interrogation room. Law \& Human Behavior, 23, 499-516.

47. Bartlett, M. S., Littlewort, G., Frank, M. G., Lainscsek, C., Fasel, I., \& Movellan, J. (2006). Fully automatic facial action recognition in spontaneous behavior. Journal of Multimedia, 6, 22-35.

48. Collins, R., Lincoln, R., \& Frank, M.G. (2002). The effect of rapport in forensic interviewing. Psychiatry, Psychology, \& Law, 9, 69-78.

\section{Further reading list}

Enter your "Further Reading" list here. This section will contain additional recommended readings (books, articles, websites, etc.) that are not included in the reference section. For some articles, this section may include references for specific techniques or protocols; for others, a listing of book chapters that provide general information on the topic may be sufficient. References in the reading list are not numbered, but otherwise should follow the style outlined for the References section.

\section{Cross-references}

See: Social and psychological aspects;

Terrorism risk: characteristics and features; 


\section{Deterrence;}

Physical security; models and countermeasures

\section{Glossary terms}

\section{Malfeasance}

Hot Spots

Micro Expression

Content Based Criteria Analysis

Demeanor

Pinocchio response

Meta-Analysis 